\title{
Nonlinear transverse vibrations of clamped beams carrying two or three concentrated masses at various locations
}

\author{
A.Rahmouni ${ }^{1,2}$ and R. Benamar ${ }^{1}$ \\ ${ }^{1}$ Laboratoire des Etudes en Simulation, Instrumentation et Mesures, EMI, Mohammed V University, Rabat, Maroc \\ ${ }^{2}$ Laboratoire Mécanique Productique et Génie Industriel, EST, University Hassan II-Casablanca, Casablanca, Maroc
}

\begin{abstract}
In a recent work, a discrete model for geometrically nonlinear transverse free constrained vibrations of beams with various end conditions has been developed and validated via comparison with known results corresponding to nonlinear vibration of clamped beams carrying a concentrated mass. It is extended here to continuous beams carrying two or three concentrated masses at various locations and subjected to large vibration amplitudes. The discrete model used is an $N$-dof ( $N$-Degrees of Freedom) system made of $N$ masses placed at the ends of solid bars connected by springs, presenting the beam flexural rigidity. The large transverse displacements of the bar ends induce a variation in their lengths giving rise to axial forces modelled by longitudinal springs causing nonlinearity. The calculations made allowed application of the semi-analytical model developed previously for nonlinear structural vibration involving three tensors, namely the mass tensor $\mathrm{m}_{\mathrm{ij}}$, the linear rigidity tensor $\mathrm{k}_{\mathrm{ij}}$ and the nonlinearity tensor $\mathrm{b}_{\mathrm{ijkl}}$ presenting the effect of the change in the bar lengths. The addition of three concentrated masses studied here induces a change in the mass matrix. By application of Hamilton's principle and spectral analysis in the modal basis, the nonlinear vibration problem is reduced to a nonlinear algebraic system, using an explicit method, developed previously for non-linear structural vibration. This study shows that concentrated masses may be used for practical purposes to shift the resonant frequency; if the three masses locations are appropriately chosen.
\end{abstract}

\section{Introduction}

Application of the discrete model developed in the work $[1,2,3]$ is made here to a Bernoulli beam carrying two or three concentrated masses at various locations and subject to geometrical nonlinear vibration due to large transverse displacements. This model focuses on the known physical phenomenon of the dynamic behavior: the stretching of the beam induces nonlinearity. To escape the occurrence of modal coupling, internal resonance, bifurcation points and chaos, the amplitudes considered here do not exceed the radius of gyration $r$ of the cross section of the beam with respect to the neutral axis given by $r=\sqrt{I / S}$, where $S$ is the beam section area and $I$ is the quadratic moment of the beam with respect to the neutral fiber (in the case of a rectangular section, $r$ is equal to the thickness of the beam divided by $\sqrt{12}$ ). This study shows that the developed model may be used to study successfully non-linear vibrations of beams carrying many concentrated masses simply by changing the mass matrix but without any change in the linear and nonlinear stiffness tensors, corresponding to a uniform beam defined in [1].
In addition, this discrete model may be very easily adapted to the study of beams with variable cross sections, with concentrated masses or stiffness, or with discontinuities in the section, the stiffness or the material properties.

\section{Presentation and nomenclature}

The studied model of a beam with three concentrate dmasses $M_{s}, M_{t}$ and $M_{q}$ is shown in Figure 1:

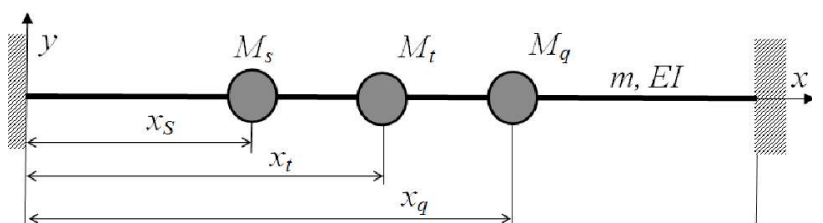

Fig. 1: Clamped beam with three concentrated masses $M_{s}, M_{t}$ and $M_{q}$

Figure 2 shows the discrete system with $N$-dof considered in the present application, consisting on $N$ masses $m_{l}, \ldots$, $\mathrm{M}_{\mathrm{s}}+m_{s-1}, \ldots, M_{t}+m_{t-1}, \ldots, M_{q}+m_{q-1}, \quad \ldots, m_{N}$ connected 
by $N+2$ torsional springs and $N+2$ longitudinal tension springs, considered in its neutral position.

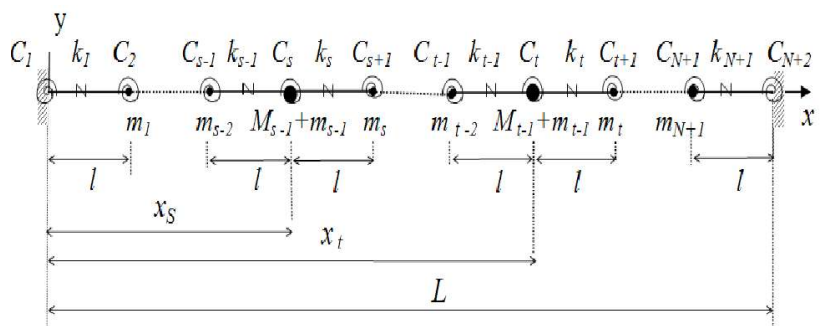

Fig. 2: Discrete system with several degrees of freedom $(N-$

dof), modelling a clamped beam with two concentrated masses

$$
M_{s} \text { and } M_{t}
$$

where the stiffness of the torsionals springs representing the flexural rigidity of the beam is $C_{i}=\frac{E I}{l}$. Equation (1) determines the location coordinates of the three masses:

$i=\frac{{ }^{x}}{l}+1$ for $s, t$ and $q$

$N$ must be a common multiple of $s, t$ and $q$.

\section{Dimensionless formulations}

The following equations link the dimensional values to the dimensionless ones (with an asterisk):

$$
\begin{aligned}
& y^{*}=\frac{y}{r} \\
& \omega^{*}=\frac{\omega L^{2}}{\sqrt{\frac{E I}{\rho S}}}
\end{aligned}
$$

$$
\eta_{i}=\frac{{ }^{x}}{L} \quad i=s \text { ou } t
$$

$\alpha_{i}=\frac{\text { concentrated mass }}{\text { total mass of the beam }}=\frac{M_{i}}{m}=\frac{M_{i}}{\rho S L} \quad i=s$ ou $t$

where $\rho$ is the density of the beam density, E the Young's modulus, $\alpha_{\mathrm{i}}$ le ratio of the concentrated mass $i$ to the total mass of the beam, and $\eta_{i}$ the non-dimensional location of the concentrated mass $i$.

\section{General form of the masses matrix in the case of a beam with three concentrated masses}

The presence of the three masses in the nodes $s, t$ and $q$ of the beam induces changes of the $s^{\text {th }}, t^{\text {th }}$ and $q^{\text {th }}$ diagonal elements of the mass matrix. The matrix $\left[m_{i j}\right]$ of order $N-2$ has the form [2]:

$$
\left[m_{i j}\right]=\frac{\rho S L}{(N+1)}\left(\begin{array}{cccccccc}
1 & 0 & 0 & \ldots & & \ldots & 0 & 0 \\
0 & 1 & \ldots & & 0 & & & 0 \\
0 & \ldots & 0 & \ldots & & & \\
\ldots & & \left(1+(N+1) \alpha_{S}\right) & \ldots & & & \ldots \\
0 & \ldots & 0 & 0 & & & 0 \\
\ldots & & \ldots & \left(1+(N+1) \alpha_{t}\right) & & & 0 \\
& & & \ldots & 0 & \ldots & 0 \\
0 & \ldots & & & \left.1+(N+1) \alpha_{q}\right) & 0 & 0 \\
& & & & 0 & 1 & 0 \\
0 & 0 & \ldots & & & \ldots & 0 & 1
\end{array}\right)
$$

\section{General form of the linear stiffness matrix $\left[k_{i j}\right]$}

We consider the case where the masses do not change the bending stiffness. The general form of the linear stiffness matrix $\left[k_{i j}\right]$ to take into account (of order $N-2$ ) is unchanged, compared to that of a uniform beam. In the case of a beam carrying masses it can be written as follows [2]:

$$
\left[\mathbf{K}_{\mathrm{cc}(N-2)}\right]=\frac{(N+1)^{3} E I}{L^{3}}\left(\begin{array}{cccccccc}
6 & -4 & 1 & 0 & . & . & . & 0 \\
-4 & 6 & -4 & 1 & 0 & . & . & . \\
1 & -4 & 6 & -4 & 1 & 0 & . & . \\
0 & . & . & . & . & . & . & . \\
. & . & . & . & . & . & . & . \\
. & . & . & . & . & . & . & . \\
. & . & . & 0 & 1 & -4 & 6 & -4 \\
. & . & . & . & 0 & 1 & -4 & 6
\end{array}\right)
$$

\section{Results in the linear case for a beam with two concentrated masses}

The results obtained for $N=49$ are given in Table 1

Table 1: The first three natural frequencies of the discrete system ( $\mathrm{N}=49)$, for $\alpha_{i}=1, \eta_{q}=0$ and different values of $\eta_{s}, \eta_{t}$

\begin{tabular}{|l|l|l|l|l|l|l|}
\hline$\eta_{s}$ & 0 & 0.1 & 0.2 & 0.3 & 0.4 & 0.5 \\
\hline$s$ & 1 & 5 & 10 & 15 & 20 & 24 \\
\hline$\eta_{t}$ & 0 & 0.9 & 0.8 & 0.7 & 0.6 & 0.5 \\
\hline$t$ & 49 & 44 & 39 & 34 & 29 & 25 \\
\hline$\omega_{\text {disc } 1}^{\mathrm{ccnl}}$ & 22.37 & 22.21 & 17.02 & 12.35 & 9.95 & 9.16 \\
\hline$\omega_{\text {disc } 2}^{\mathrm{ccnl}}$ & 64.18 & 52.10 & 31.00 & 26.43 & 33.49 & 63.11 \\
\hline$\omega_{\text {disc } 3}^{\mathrm{ccnl}}$ & 125.65 & 78.65 & 64.81 & 99.52 & 115.53 & 94.97 \\
\hline
\end{tabular}

It is noted that when the masses are placed in the middle of the beam, the first natural frequency decreases; this is due to the shape of the first vibration mode which has a maximum amplitude in the middle of the beam, while the second eigenmode remains unchanged at this location 
since the form of this mode has a node in the middle of the beam. Note also that when the masses are placed in $1 / 4$ of the beam span, the second natural frequency decreases until the value of the first natural frequency (for

$\eta_{s}=0,3 ; \eta_{t}=0,7$ and $\eta_{q}=0$; this is due to the shape of the second vibration mode having three antinodes at these locations of the beam.

\section{Cases of non-linear vibration of a beam carrying two or three concentrated masses}

It should be noted that the coefficients of linear rigidity and non-linear tensors $\bar{k}_{i j}$ and $\bar{b}_{i j k l}$ calculated in the modal basis are different from those of a continuous beam, because the transition matrix $[\boldsymbol{\Phi}]$ has undergone a change, due to addiction of concentrated masses, as illustrated in the following equations.

$$
\begin{aligned}
& \bar{m}_{i j}=\Phi_{s i} \Phi_{t j} m_{s t} \quad i, j=1, \ldots, N \\
& \bar{k}_{i j}=\varphi_{s i} \varphi_{t j} k_{s t} \quad i, j=1, \ldots, N \\
& \bar{b}_{i j k l}=\varphi_{s i} \varphi_{t j} \varphi_{p k} \varphi_{q l} b_{s t p q} \quad i, j, k, l=1, \ldots, N
\end{aligned}
$$

In the displacement basis, the nonlinear system is written as:

$$
[\mathbf{M}]\{\ddot{\mathbf{y}}\}+[\mathbf{K}]\{\mathbf{y}\}+\frac{3}{2}[\mathbf{B}(y)]\{\mathbf{y}\}=\{\mathbf{0}\}
$$

where $[\mathrm{M}],[\mathrm{K}]$, and $[\mathrm{B}(\mathrm{y})]$ are respectively the matrix mass, the linear matrix rigidity, and the nonlinear rigidity tensor. It should be noted here that Equation (11) becomes, if the non-linear term is neglected, identical to the classical equation of linear modal analysis:

$$
[\mathbf{M}]\{\ddot{\mathbf{y}}\}+[\mathbf{K}]\{\mathbf{y}\}=\{\mathbf{0}\}
$$

The tonsorial form of the non linear energy $V_{\mathrm{nl}}$ is:

$$
V_{\mathrm{nl}}=\frac{1}{2} y_{i} y_{j}^{y} k^{y} l_{i j k l}
$$

Using the Pythagorean Theorem, we express the nonlinear energy versus the characteristics of the beam [1], see Figure3:

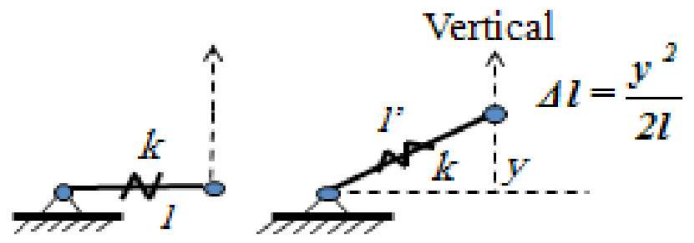

Fig. 3: modelling the tensile bars rigidity $k=E S / l$ and calculating the nonlinear energy with neglecting horizontal displacements
Using the known formula $V_{n l}=\frac{1}{2} k(\Delta l)^{2}$, the nonlinear potential energy is deduced as $V_{n l} \cong \frac{k}{8 l^{2}} y^{4}$.

By the same approach, we calculate the nonlinear energy for all of the bars (see Figure 4), which we compare to the tonsorial expression to determine the coefficients of the nonlinear stiffness tensor [B $(y)]$ [1] (see equations (14) to (17)).

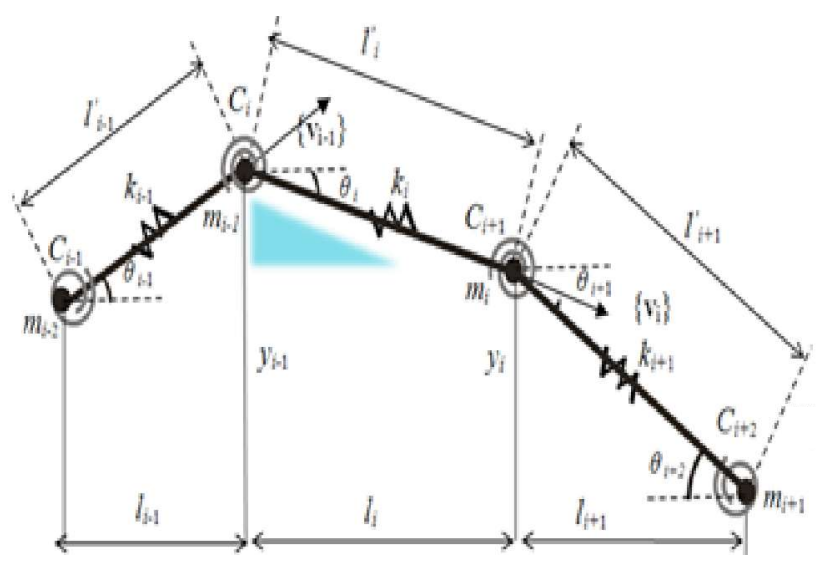

Fig. 4 Discrete system zoomed in the neighbourhood of bar $i$

$$
\begin{aligned}
& b_{i i i i} \cong 2 \frac{E S}{8 l^{3}} \cong 2(N+1)^{3} \frac{E S}{8 L^{3}} \quad i=1, \ldots, N \\
& b_{i(i-1)(i-1)(i-1)}=b_{(i-1) i(i-1)(i-1)}= \\
& b_{(i-1)(i-1) i(i-1)}=b_{(i-1)(i-1)(i-1) i}=\frac{E S}{8 l^{3}} \quad i=2, \ldots, N \\
& b_{i(i-1)(i-1) i}=b_{(i-1) i(i-1) i}=b_{(i-1)(i-1) i i}=b_{(i-1) i i}(i-1) \\
& =b_{i(i-1) i(i-1)}=b_{i i(}(i-1)(i-1) \cong \frac{E S}{8 l^{3}} i=2, \ldots ., N \\
& b_{i i i(}(i-1)=b_{i i(}(i-1) i=b_{i(i-1) i i}=b_{(i-1) i i i} \cong-\frac{E S}{8 l^{3}} \quad i=2, \ldots, N
\end{aligned}
$$

The other coefficients are equal to zero.

Our method consists on applying Hamilton's principle in the modal basis:

$$
\delta \int_{0}^{2 \pi / \omega_{\mathrm{disc}}^{\mathrm{nl}}}(V-T) \mathrm{dt}=0
$$

We obtain a system of nonlinear algebraic equations in which time is not involved:

$$
3 a_{i} a_{j} a_{k} \bar{b}_{i j k r}+2 a_{i} \bar{k}_{i r}-2 a_{i}\left(\omega_{\mathrm{disc}}^{\mathrm{nl}}\right)^{2} \bar{m}_{i r}=0 \quad i, j, k, r=1, N
$$

We used the explicit method presented in [4] in the Modal basis to solve this equation: The explicit formulation is based on an approximation which consists 
on assuming, when the first nonlinear mode shape is under examination, that the contribution vector $\{\mathbf{a}\}^{T}=\left[a_{1} a_{2} \ldots a_{N}\right]$ can be written as $\{\mathbf{a}\}^{T}=\left[a_{1} \varepsilon_{2} \ldots \varepsilon_{i} \ldots \varepsilon_{N}\right]$ with $\varepsilon_{i}$ being small compared to $a_{1}\left(a_{1}^{2} \varepsilon_{v}=0 \quad \varepsilon_{i} \varepsilon_{j} \varepsilon_{k} \approx 0 \quad a_{1} \varepsilon_{i} \varepsilon_{j} \approx 0\right)$. Neglecting in the expression $a_{i} a_{j} a_{k} \bar{b}_{i j k r}$ of Eq. (19), which involves summation for the repeated indices $\mathrm{i}, \mathrm{j}$, $\mathrm{k}$ the first, second and third-order terms so that the only remaining term $a_{1}^{3} \bar{b}_{111 r}$, leads to:

$$
\left({\overline{k_{j r}}}_{-}-\left(\omega_{\mathrm{disc}}^{\mathrm{nl}}\right)^{2} \bar{m}_{j r}\right) \varepsilon_{j}+\frac{3}{2} a_{1}^{3} \bar{b}_{111 r}=0 \quad \text { for } r=2, \ldots, N
$$

The contributions $\left\{a_{1 \text { disc }} \varepsilon_{1} \varepsilon_{2} \ldots \varepsilon_{N}\right\}$ in the modal basis are calculated by [1]:

$\varepsilon_{\mathrm{r}}=\frac{\frac{3}{2} \mathrm{a} \frac{3}{\mathrm{~b}_{111 \mathrm{r}}}}{\left(\left(\omega_{\mathrm{disc}}^{\mathrm{nl}}\right)^{2} \overline{\mathrm{m}_{\mathrm{rr}}}-\overline{\mathrm{k}_{\mathrm{rr}}}\right)} \quad$ for $\quad \mathrm{r}=2, \ldots ., \mathrm{N}$

The non linear frequency is calculated by:

$\left(\omega_{\mathrm{d} \text { isc }}\right)^{2}=\frac{\overline{k_{11}}}{m_{11}}+\frac{3}{2} \frac{\overline{b_{1111}}}{m_{11}} a_{1}^{2}$

After solving the equation in the modal basis, the amplitudes are calculated in the displacement basis:

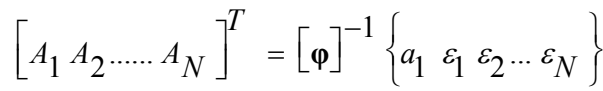

The amplitude is the maximum of $\left(A_{1} A_{2} \ldots . A_{N}\right)$, and we plot the dimensionless frequency curve as a function of the dimensionless amplitude

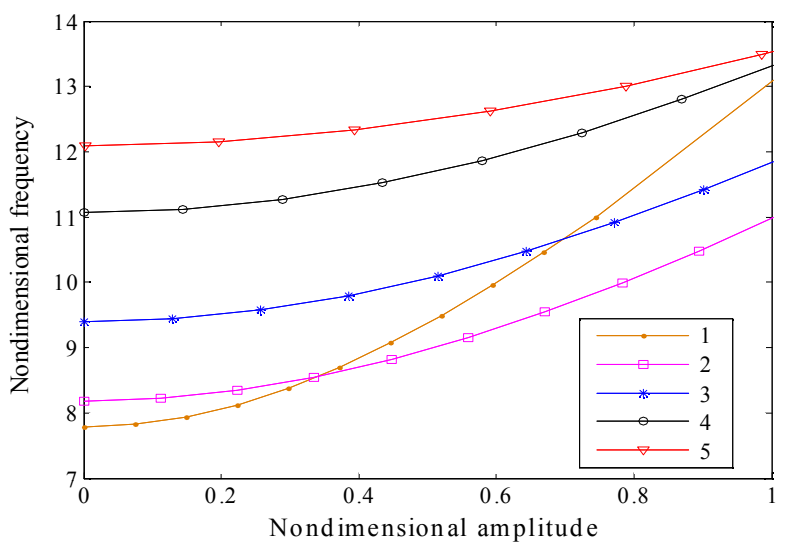

Fig. 5: Frequency curves according to the amplitude corresponding to the discrete systems with $N=49$ dof, $\alpha_{i}=1$, and $\left(1: \eta_{\mathrm{s}}=\eta_{\mathrm{t}}=\eta_{\mathrm{q}}=0.5 ; 2: \eta_{\mathrm{s}}=0.4, \eta_{\mathrm{t}}=0.5\right.$ and $\eta_{\mathrm{q}}=0.6$;

3: $\eta_{\mathrm{s}}=0.3, \eta_{\mathrm{t}}=0.5$ and $\eta_{\mathrm{q}}=0.7 ; 4: \eta_{\mathrm{s}}=0.2, \eta_{\mathrm{t}}=0.5$ and $\eta_{\mathrm{q}}=0.8$; 5: $\eta_{\mathrm{s}}=0.1, \eta_{\mathrm{t}}=0.5$ and $\eta_{\mathrm{q}}=0.9$ )
We note that when the three masses are in the middle of the beam, stretching are important which increases the nonlinearity.

\section{Conclusions}

The discrete model developed and validated in the case of a continuous beam presented in $[1,2,3]$, was applied to beams with two or three concentrated masses. Linear and nonlinear vibrations were examined. This shows the effectiveness of this discrete model, its formulation and the associated program for the study of linear and nonlinear vibrations of a beam with discontinuities in the distribution of masses. The concentrated masses change significantly the beam dynamic response.

\section{References}

1. A. Rahmouni, Z. Beidouri and R. Benamar, $A$ discrete model for geometrically nonlinear transverse free constrained vibrations of beams with various end conditions, Journal of Sound and Vibration 332 (2013) 5115-5134.

2. A. Rahmouni, Z. Beidouri and R. Benamar, $A$ discrete model for the natural frequency and mode shapes of beams with various boundary conditions MATEC Web of Conferences 01(2012); 1.DOI: 10.1051/matecconf $/ 20120110015$

3. A. Rahmouni and R. Benamar $A$ discrete model for geometrically nonlinear transverse free constrained vibrations of clamped beam carrying a concentrated mass at various locations, EURODYN (2014) ISSN 2311-9020 ISBN 978-972-752-165-4, 2093-2099

4. M. El. Kadiri, R. Benamar, R.G. White, Improvement of the semi-analytical method, based on Hamilton's principle and spectral analysis, for determination of the geometrically non-linear free response of thin straight structures. Part I: application to $C-C$ and $S S-C$ beams, Journal of Sound and Vibration, 249(2002) 263-305. 